Disclosure of Interests: None declared DOI: 10.1136/annrheumdis-2021-eular.857

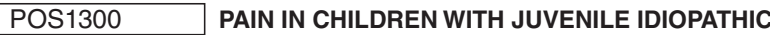 ARTHRITIS TREATED TO TARGET: 24 MONTH RESULTS FROM A RANDOMISED TRIAL}

K. Spekking ${ }^{1}$, P. De Boer ${ }^{1}$, S. A. Bergstra ${ }^{2}$, J. M. Van den Berg ${ }^{3}$, D. Schonenberg-Meinema ${ }^{3}$, L. W. A. Van Suijlekom-Smit ${ }^{4}$, M. Van Rossum ${ }^{5,6}$ Y. Koopman-Keemink ${ }^{7}$, R. Ten Cate ${ }^{1}$, C. Allaart ${ }^{2}$, D. M. C. Brinkman ${ }^{1}$, P. C. E. Hissink Muller'. ' 'Leiden University Medical Center, Department of Pediatric Rheumatology, Leiden, Netherlands; ' ${ }^{2}$ Leiden University Medical Center, Department of Rheumatology, Leiden, Netherlands; ${ }^{3} E m m a$ Children's Hospital, Amsterdam University Medical Centers, Department of Pediatric, Immunology, Rheumatology and Infectious Diseases, Amsterdam, Netherlands; ${ }^{4}$ Sophia Children's Hospital Erasmus Medical Center, Department of Pediatrics/ Pediatric Rheumatology, Rotterdam, Netherlands; ${ }^{5}$ Emma Children's Hospital, Amsterdam University Medical Centers, Department of Pediatrics, Amsterdam, Netherlands; ${ }^{6}$ Amsterdam Rheumatology and Immunology Center | Reade, Department of Pediatric Rheumatology, Amsterdam, Netherlands; ${ }^{7}$ Hagaziekenhuis Juliana Children's Hospital, Department of Pediatrics, the Hague, Netherlands

Background: Juvenile Idiopathic Arthritis (JIA) is the most common auto-immune disease in children and pain is a common symptom. Despite treatment advances in recent years, pain often persists when the disease is inactive. Treating JIA to target has been widely recommended ${ }^{1}$, but effectiveness in pain reduction has not been proven yet ${ }^{2}$.

Objectives: To compare pain scores over two years in three treatment arms in JIA patients that were treated to target and to identify baseline characteristics predicting unfavorable pain outcomes.

Methods: DMARD naïve children who participated in the BeSt for kids study ${ }^{3}$ with oligoarticular JIA, RF negative polyarticular JIA and juvenile psoriatic arthritis were included. Patient were treated to target aimed at inactive disease according to 1 of 3 different treatment groups; 1 ) initial sequential DMARD monotherapy 2) initial methotrexate (MTX) with prednisolone bridging or 3) initial MTX with etanercept. Pain intensity was measured using a $100 \mathrm{~mm}$ Visual Analogue Scale during 24 months of follow up with 3-monthly intervals. Potential differences in VAS pain scores over time between treatment arms were compared using linear mixed models with random intercept and random slope for visits clustered within patients. A similar multivariable mixed model was used to assess the ability of several baseline characteristics to predict the chance of high pain levels during follow-up.

Results: 92 patients were randomized into the three treatment groups. Overall, pain scores over time reduced from mean 55.3 (SD 21.7) at baseline to 19.5 (SD 25.3) after 24 months. When comparing pain over time per arm, pain scores decreased significantly over time $\beta-1.37$ (95\% Cl $-1.726 ;-1.022)$. No significant difference was found in pain over time between treatment groups (interaction term treatment arm*time (months) $\beta(95 \% \mathrm{Cl})$ arm $10.13(-0.36$; $0.62)$ and arm $20.37(-0.12 ; 0.86)$ compared to arm 3), Figure 1. Correction for sex and symptom duration as possible confounders yielded similar results. Multiple baseline characteristics demonstrated a significant predictive value for pain over time: VAS pain (scale $0-100)$ with $\beta 0.44$ (95\% Cl 0.25; 0.65), VAS physician (scale $0-100)$ with $\beta-0.34(-0.55 ;-0.06)$, number of active joints with $\beta 0.77(0.19 ; 1.34)$, Child Health Questionnaire Physical summary Score with $\beta-0.42(-0.72 ;-0.11)$ and Psychosocial summary Score $\beta-0.42(-0.77 ;-0.06)$. Symptom duration, VAS patient/parent and NSAID use were no significant predictors, Table 1.

Conclusion: Treatment to target seems effective in pain reduction in non-systemic JIA patients irrespective of initial treatment strategy. Several baseline predictors for pain over time were identified, which could serve as an initial indication for non-systemic JIA-patients with a high risk of pain despite a strict treat-totarget strategy.

REFERENCES:

[1] Ravelli A, Consolaro A, Horneff G, et al. Treating juvenile idiopathic arthritis to target: recommendations of an international task force. Ann Rheum Dis. 2018;77(6):819-828.

[2] Schoemaker, C.G., Swart, J.F. \& Wulffraat, N.M. Treating juvenile idiopathic arthritis to target: what is the optimal target definition to reach all goals? Pediatr Rheumatol. 18, 34 (2020)

[3] Hissink Muller P, Brinkman DMC, Schonenberg-Meinema D, et al. Treat to target (drug-free) inactive disease in DMARD-naive juvenile idiopathic arthritis: 24-month clinical outcomes of a three-armed randomised trial. Ann Rheum Dis. 2019 Jan;78(1):51-59.

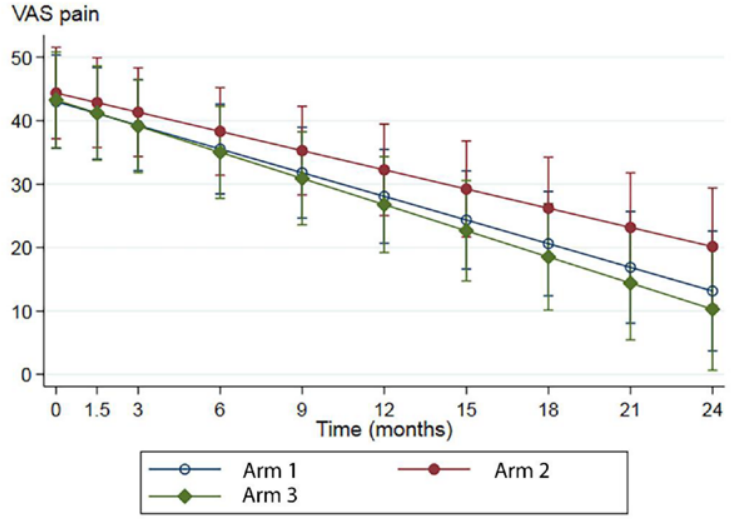

Figure 1 | VAS pain over time. Linear mixed models with random intercept and random slope for visits clustered within patients. VAS pain measured on a $100 \mathrm{~mm}$ scale. Error bars indicate $95 \%$ confidence intervals.

Table 1. Baseline Characteristics

\begin{tabular}{llll}
\hline & $\beta$ & p-value & 95\% Confidence Interval \\
\hline VAS Pain & 0.45 & 0.000 & 0.25 to 0.65 \\
VAS Physician & -0.31 & 0.014 & -0.55 to -0.06 \\
VAS Patient/parent & -0.02 & 0.873 & -0.21 to 0.18 \\
No. of active joints & 0.77 & 0.009 & 0.19 to 1.34 \\
PhS $^{\star}$ & -0.42 & 0.008 & -0.72 to -0.11 \\
PsS $^{\star *}$ & -0.42 & 0.022 & -0.77 to -0.06 \\
Symptom duration (mo.) & 8.09 & 0.118 & -2.08 to 18.26 \\
NSAID use & -1.30 & 0.791 & -10.91 to 8.31
\end{tabular}

VAS Pain, VAS physician and VAS patient/parent was measures on a $100 \mathrm{~mm}$ scale. ${ }^{*} \mathrm{PhS}$ Physical Summary Score of the Child Health Questionnaire Parent form 50 (scale $0-100) .{ }^{* *} P s S=$ Psychosocial Summary Score of the CHQ-PF50 (scale 0-100).

Disclosure of Interests: None declared

DOI: 10.1136/annrheumdis-2021-eular.988

\section{POS1301 DRUG SURVIVAL OF BIOLOGICS WITH RESPECT TO COMBINATION WITH METHOTREXATE IN TREATMENT OF POLYARTICULAR JIA}

G. Horneff ${ }^{1}$, D. Windschall ${ }^{2}$, K. Minden ${ }^{3}$, T. Hospach ${ }^{4}$, F. Dressler ${ }^{5}$, F. WellerHeinemann $^{6}$, B. Huegle ${ }^{7}$, I. Foeldvari ${ }^{8}$, A. Klein ${ }^{1}$ on behalf of BIKER registry. ${ }^{1}$ Asklepios Clinic, Paediatics, Sankt Augustin, Germany; ${ }^{2}$ St. Josef Stift, Paediatric Rheumatology, Sendenhorst, Germany; ${ }^{3}$ Deutsches RheumaForschungszentrum Berlin (DRFZ), ein Institut der Leibniz-Gemeinschaft, Epidemiology, Berlin, Germany; ${ }^{4}$ Klinikum Stuttgart - Olga Hospital / Women's Hospital, Paediatrics, Stuttgart, Germany; ${ }^{5} \mathrm{MHH}$ Kinderklinik Station 61b, Paediatrics, Hannover, Germany; ${ }^{6}$ Prof Hess Childrens Hospital, Paediatrics, Bremen, Germany; ${ }^{7}$ Deutsche Zentrum, Kinder- und Jugendrheumatologie, Garmisch-Partenkirchen, Germany; ${ }^{8}$ Zentrum, Kinderheumatologie, Hamburg, Germany

Background: In polyarticular juvenile idiopathic arthritis (pJIA) biologic therapies are often combined with methotrexate (MTX). This combination was shown to increase efficacy in adult rheumatoid arthritis patients. MTX may also have a protective effect on the formation of anti-drug antibodies and thus may prolong drug survival. In pJIA, there are few and sometimes controversial data available. Objectives: To compare the effect of combination treatment with MTX on discontinuation due to inefficacy and on drug survival discontinuation of biologics approved for first line treatment of pJIA.

Methods: Patients from the German BIKER registry with their first treatment course with Adalimumab, Etanercept, Golimumab or Tocilizumab were selected. Rates of ineffectiveness-related withdrawal were analysed and compared using $\chi 2$-test, Wald-test and Kaplan-Meier analysis of patients receiving biologic monotherapy or concomitant methotrexate. Cases were censored if MTX was discontinued before the biologic.

Results: 2173 pJIA patients were identified who for the first time received a biologic. Etanercept (ETA) was by far the most frequently used biologic for first line biologic treatment in pJIA (77\%) followed by Adalimumab (ADA, 16\%). Patients on Golimumab (GOL) received most frequently a combination with MTX (86.5\%), while patients on Tocilizumab (TOC) had the lowest rate of combination treatment (53\%).

ETA/ADA/GOL/TOC was given as monotherapy in 500(30\%)/89(26\%)/5(13.5\% )$/ 46(47 \%)$ and combined with MTX in $1179(70 \%) / 259(74 \%) / 32(86.5 \%) / 51(53$ 